\title{
GRAPHIC SPLITTING OF COGRAPHIC MATROIDS
}

\author{
NAIYER PIROUZ \\ Department of Mathematics, University of Pune \\ Pune - 411007, India \\ e-mail: naiyer.pirouz@gmail.com
}

\begin{abstract}
In this paper, we obtain a forbidden minor characterization of a cographic matroid $M$ for which the splitting matroid $M_{x, y}$ is graphic for every pair $x, y$ of elements of $M$.
\end{abstract}

Keywords: binary matroid, graphic matroid, cographic matroid, minor, splitting operation.

2010 Mathematics Subject Classification: 05B35, 05C50, 05C83.

\section{REFERENCES}

[1] Y.M. Borse, Forbidden-minors for splitting binary gammoids, Australas. J. Combin. 46 (2010) 307-314.

[2] Y.M. Borse, M.M. Shikare and K.V. Dalvi, Excluded-minors for the class of cographic splitting matroids, Ars Combin. 115 (2014) 219-237.

[3] H. Fleischner, Eulerian Graphs and Related Topics (North Holland, Amsterdam, 1990).

[4] F. Harary, Graph Theory (Addison-Wesley, 1969).

[5] A. Mills, On the cocircuits of a splitting matroid, Ars Combin. 89 (2008) 243-253.

[6] J.G. Oxley, Matroid Theory (Oxford University Press, Oxford, 1992).

[7] T.T. Raghunathan, M.M. Shikare and B.N. Waphare, Splitting in a binary matroid, Discrete Math. 184 (1998) 267-271. doi:10.1016/S0012-365X(97)00202-1

[8] M.M. Shikare, Splitting lemma for binary matroids, Southeast Asian Bull. Math. 32 (2007) 151-159.

[9] M.M. Shikare and G. Azadi, Determination of the bases of a splitting matroid, European J. Combin. 24 (2003) 45-52.

doi:10.1016/S0195-6698(02)00135-X 
[10] M.M. Shikare and B.N. Waphare, Excluded-minors for the class of graphic splitting matroids, Ars Combin. 97 (2010) 111-127.

[11] D.J.A. Welsh, Matroid Theory (Academic Press, London, 1976).

Received 4 March 2013

Revised 7 February 2014

Accepted 7 February 2014 